\title{
Electromagnetic response from multilayer composite coatings based on carbonyl iron, ferroelectrics and MWCNTs at microwave
}

\author{
Grigoriy E. Kuleshov ${ }^{1, *}$, Alexey V. Sbrodov ${ }^{1}$, and Tatyana N. Shematilo ${ }^{1}$ \\ ${ }^{1}$ National Research Tomsk State University, 634050, Tomsk, Russia
}

\begin{abstract}
The electromagnetic characteristics of composite materials based on carbonyl iron, ferroelectrics, and MWCNTs were studied in this article. The complex magnetic and dielectric constants of the experimental samples were measured in the frequency range from $1 \mathrm{GHz}$ to $18 \mathrm{GHz}$. The results of calculation and the experimental study of the frequency dependences of the reflection coefficient on a multilayer composite coating located on a reflective surface are presented.
\end{abstract}

\section{Introduction}

Today, widespread use of high-frequency electronic equipment in all areas of human life is noted. These are wireless data receivers and transmitters (Bluetooth, Wi-Fi devices, cell phones), various household appliances, sensors, IoT devices, medical equipment. Nevertheless, the use of the aforementioned technique creates a number of negative factors. There are problems of electromagnetic safety when interception of confidential or secret information is carried out and also problems of electromagnetic compatibility, i.e. mutual interference by neighboring radio equipment elements [1]. To reduce the negative effects of electromagnetic radiation, radar absorbing materials are used. Ferrites [2], carbonyl iron (CI) [3], carbon nanotubes [4] and multiferoics [5] have proven themselves well in the microwave range. However, at the moment there is no thin and light single-component material that would have good absorbing properties in a wide frequency band. Multicomponent composites or multilayer coatings are used for these purposes [6].

In this work, we study the electromagnetic response from multicomponent multilayer composites based on carbonyl iron with a ferroelectric and multi-walled carbon nanotubes (MWCNT).

\section{Objects of research}

The objects of study are multicomponent composite radio materials. For the manufacture of experimental samples of composites, an EDP-20 brand epoxy resin with a hardener polyethylene polyamine (PEPA) was chosen as a binder. Powders of various radio materials were used as fillers: 1) Carbonyl iron (CI) grade P-100; 2) Ferroelectric - barium titanate

* Corresponding author: grigorij-kge@sibmail.com 
(TB) $\mathrm{BaTiO}_{3}$, containing $97 \%$ of the main phase, with particle sizes less than 100 microns ; 3) MWCNTs with an average diameter of $9.4 \mathrm{~nm}$ with a main phase content of $98 \%$ (with concentrations of 1 and 2 wt.\%) [7].

The methodology for manufacturing composite samples is presented in Fig. 1. First, the initial components are selected, weighed on Shimadzu AUX-320 scales (accurate to $0.5 \mathrm{mg}$ ) and mixed together. Next, the resulting mixture is subjected to ultrasonic dispersion for 2 minutes at a power of 50 watts. This is used to break agglomerates of the to obtain a more uniform volume distribution of the filler in the sample. Then the mixture is poured into special PTFE molds. The polymerization of the samples is carriead for 12 hours. Then, the polymerized samples are machined until they fully comply with the dimensions of the selected measuring cell.

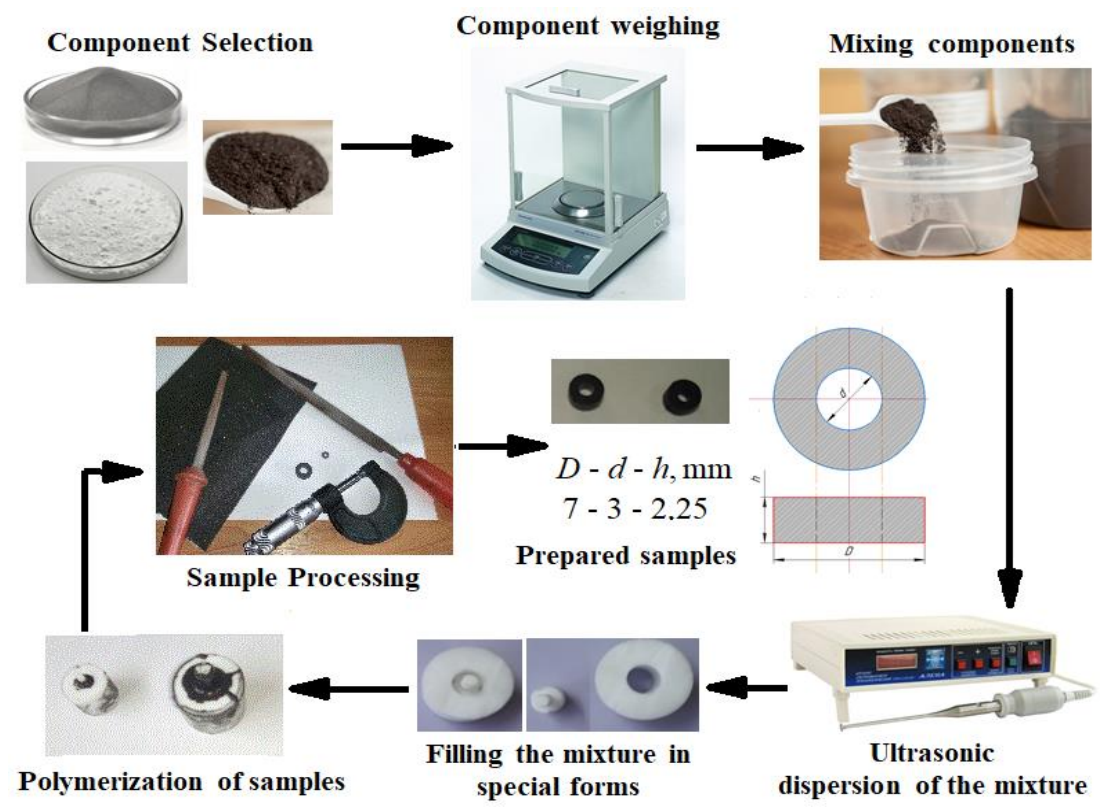

Fig. 1. Scheme of manufacturing samples of composite materials.

As a result, a number of experimental samples of single-component and multicomponent composites were obtained (Table 1).

Table 1. Manufactured samples.

\begin{tabular}{|c|c|c|c|c|}
\hline \multirow{2}{*}{$\begin{array}{c}\text { Sample } \\
\text { No. }\end{array}$} & \multirow{2}{*}{$\begin{array}{c}\text { Content of epoxy } \\
\text { resin, wt.\% }\end{array}$} & \multicolumn{3}{|c|}{ Content of the filler, wt.\% } \\
\cline { 3 - 5 } & Carbonyl iron & MWCNT & $\mathrm{BaTiO}_{3}$ \\
\hline 1 & 60 & 40 & - & - \\
\hline 2 & 40 & 60 & - & - \\
\hline 3 & 20 & 80 & - & - \\
\hline 4 & 60 & - & - & 40 \\
\hline 5 & 40 & - & - & 60 \\
\hline 6 & 20 & - & - & 80 \\
\hline 7 & 59 & 40 & 1 & - \\
\hline 8 & 58 & 40 & 2 & - \\
\hline 9 & 40 & 40 & - & 20 \\
\hline 10 & 20 & 40 & - & 40 \\
\hline
\end{tabular}




\section{Measurement technique and experimental results}

To study the frequency dependences of the electromagnetic characteristics, a Mikran P4M18 vector network analyzer was used together with a coaxial cell $\left(d_{\text {out }}=7 \mathrm{~mm}, d_{\text {in }}=3 \mathrm{~mm}\right)$. The measurements were carried out in the frequency range from 1 to $18 \mathrm{GHz}$. At the same time, several measurement schemes were implemented, they are presented in Fig. 2.
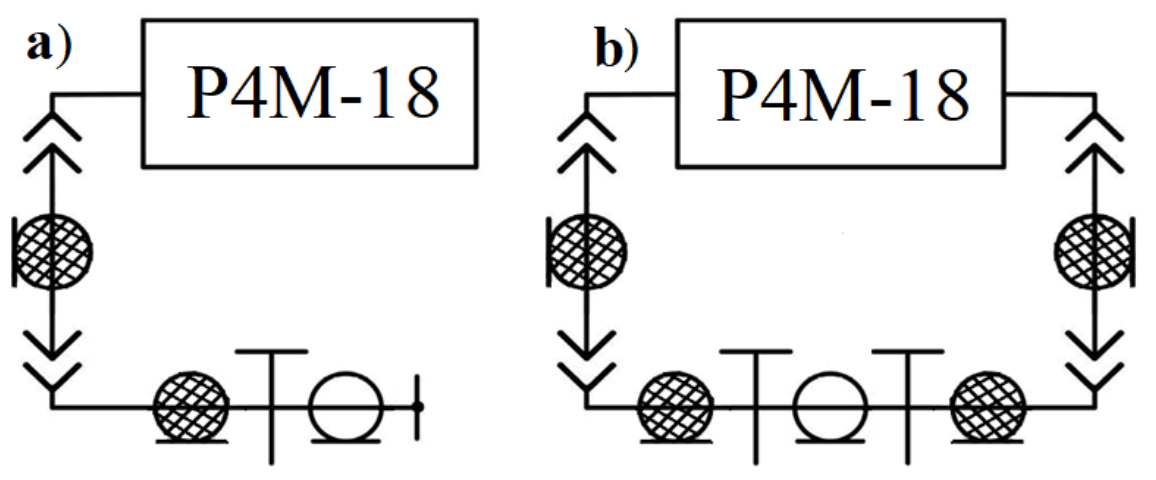

Fig. 2. Block chart of the measurement setup, "reflection" (a) and "transmission" scheme (b).

The first method (Fig. 2a) is based on direct measurement of the reflection coefficient module $(R)$ from a flat layer of magnetodielectric located on the short-circuiting in the coaxial line. It is used to study the absorbing properties of weakly reflective materials.

In the "transmission" scheme (Fig. 2b), samples are placed in the center of the coaxial cell. In this case, $S$-parameters (transmission and reflection coefficients), taking into account the change in their phase, are measured. Based on these data, it is possible to calculate the spectra of the complex permeability and the permittivity. For this purpose, the Becker-Jarvis technique [8] was used.

On the basis of the obtained spectra of the complex permeability and permittivity in the plane-wave approximation, the reflection coefficient from the layer of magnetodielectric located on the reflecting surface (metal surface) is calculated. In this case, the most interesting is the calculation of the reflection coefficient from a multilayer composite material. The plane-wave approximation and formula (1) were used for this.

$$
R=\frac{Z_{i n}^{n}-1}{Z_{i n}^{n}+1}
$$

where $Z_{i n}^{n}=\frac{Z_{i n}^{n-1}-i Z_{n} \operatorname{tg}\left(k_{n} d_{n}\right)}{Z_{n}-i Z_{i n}^{n-1} \operatorname{tg}\left(k_{n} d_{n}\right)} Z_{n}$ is input impedance of the $n$-layer at the free space, $k=\frac{2 \pi f \sqrt{\varepsilon^{*} \mu^{*}}}{c}$ is wave number, $Z_{n}=\sqrt{\frac{\mu^{\prime}+\mu^{\prime \prime}}{\varepsilon^{\prime}+\varepsilon^{\prime \prime}}}$ is wave impedance of a corresponding flat layer; $\varepsilon$ is the permittivity, $\mu$ is the permeability, $c$ is the speed of light in vacuum, $f$ is the frequency.

\section{Results and discussion}

As a result of the measurements, the frequency dependences of the complex permeability and permittivity of all the studied composite samples were obtained.

The frequency dependences of the real and imaginary parts of the complex permittivity of single-component composites are shown in Fig. 3. 

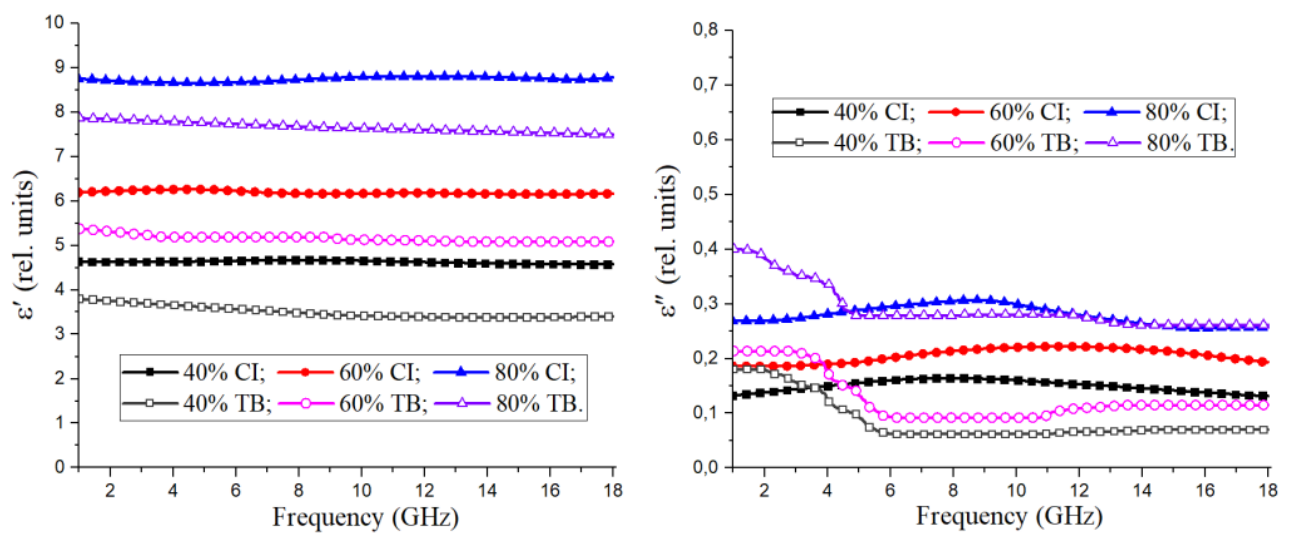

Fig. 3. Frequency dependence of the complex permittivity of a single-component composite based on carbonyl iron and barium titanate.

Analyzing the graphs you can see that at the same concentration of fillers in samples based on carbonyl iron, $\varepsilon^{\prime}$ is 1 rel.unit more than in samples based on barium titanate. With increasing concentration of fillers, the real part of the complex dielectric constant increases nonlinearly. The greater the concentration is the more significant the growth is. For all of the presented samples, $\varepsilon^{\prime}$ is practically independent of frequency.

With an increase in the concentration of fillers the values of the imaginary part of permittivity also increase. Moreover, in the beginning dielectric losses are greater for CI, then with increasing concentrations they are compared with TB. In the samples based on barium titanate in the region from 1 to $5 \mathrm{GHz}$, an anomalous dispersion of the permittivity is observed. This is possibly due to the presence of resonance of domain walls of the material at frequencies less than $1 \mathrm{GHz}$ due to particle sizes.

The frequency dependences of the complex permittivity of two-component composites are shown in Fig. 4. With the addition of multiwalled carbon nanotubes to a composite containing $40 \mathrm{wt} . \% \mathrm{CI}$, a significant increase in the values of both the real (from 4.5 to 9.5 rel. Units) and the imaginary parts of the permittivity (from 0.2 up to 1.4 rel. units) is observed. With the addition of $40 \mathrm{wt} . \% \mathrm{~TB}$, there is a significant increase in the real part of the complex permittivity (from 4.5 to 11.5 relative units) and a slight increase in the imaginary part (from 0.2 to 0.5 relative units).
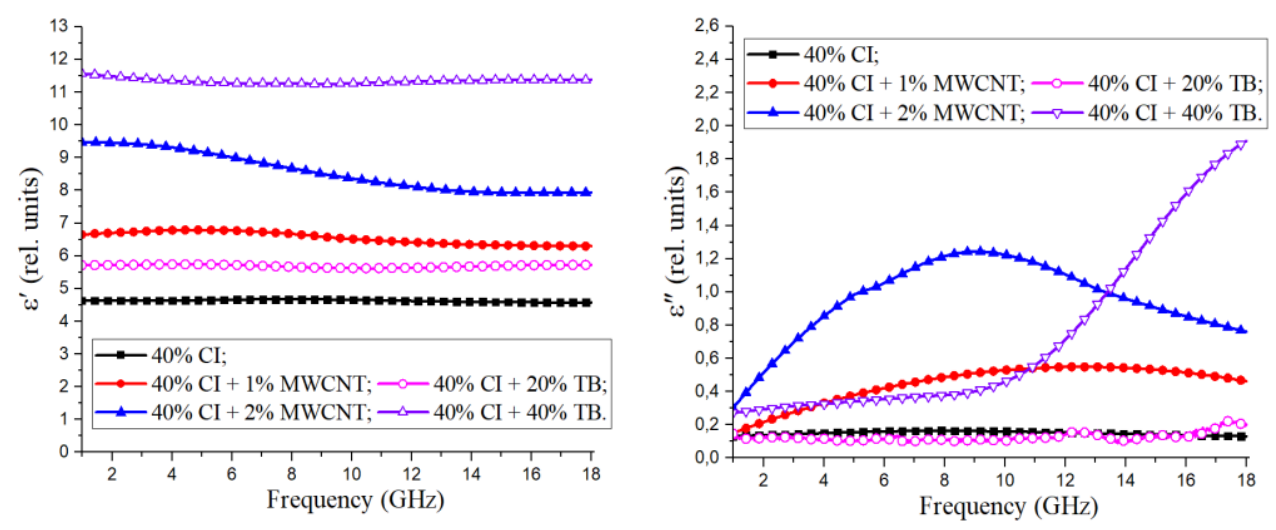

Fig. 4. Frequency dependence of the complex dielectric constant of two-component composites.

The frequency dependences of the complex magnetic permeability for all samples, except for the composite with barium titanate (non-magnetic material), are presented in Fig. 5. 

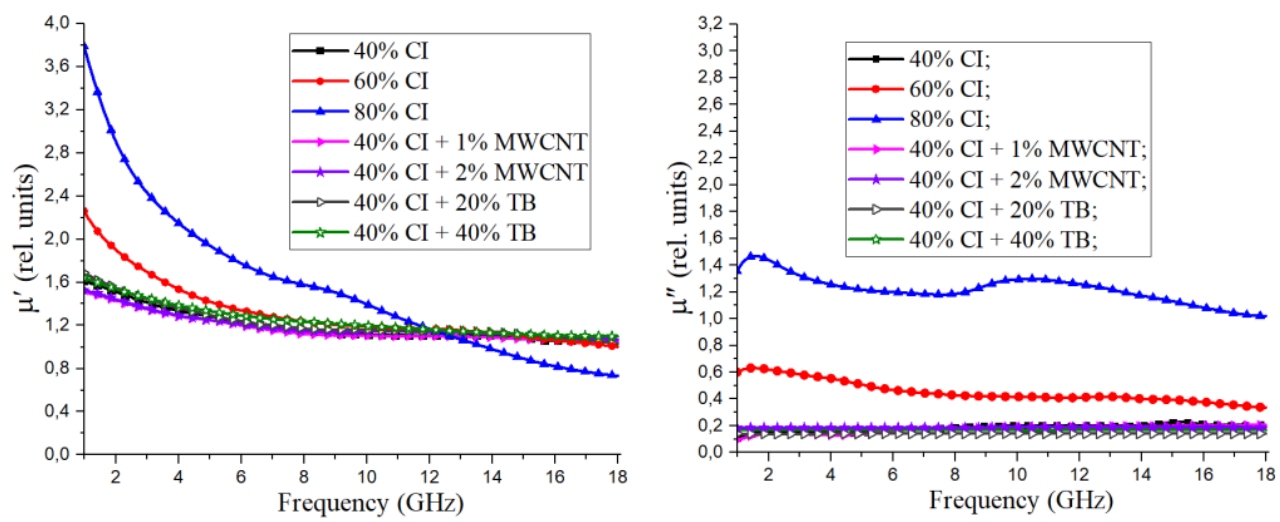

Fig. 5. Frequency dependence of the complex magnetic permeability of magnetic composites.

The graphs show that only carbonyl iron contributes to the magnetic permeability, while the remaining materials are not magnetic. For samples with a high CI content in the region of $2 \mathrm{GHz}$, the maximum of the real part and the minimum of the imaginary part of the permeability are observed. This is natural ferromagnetic resonance.

The following are the results of measurements of the electromagnetic response from multilayer composite materials located on a metal short circuit. In addition to studying the experimentally obtained dependences, a methodology for calculating the electromagnetic response from a multilayer absorbing coating located on a reflective surface was used. The results obtained are shown in Fig. 6.

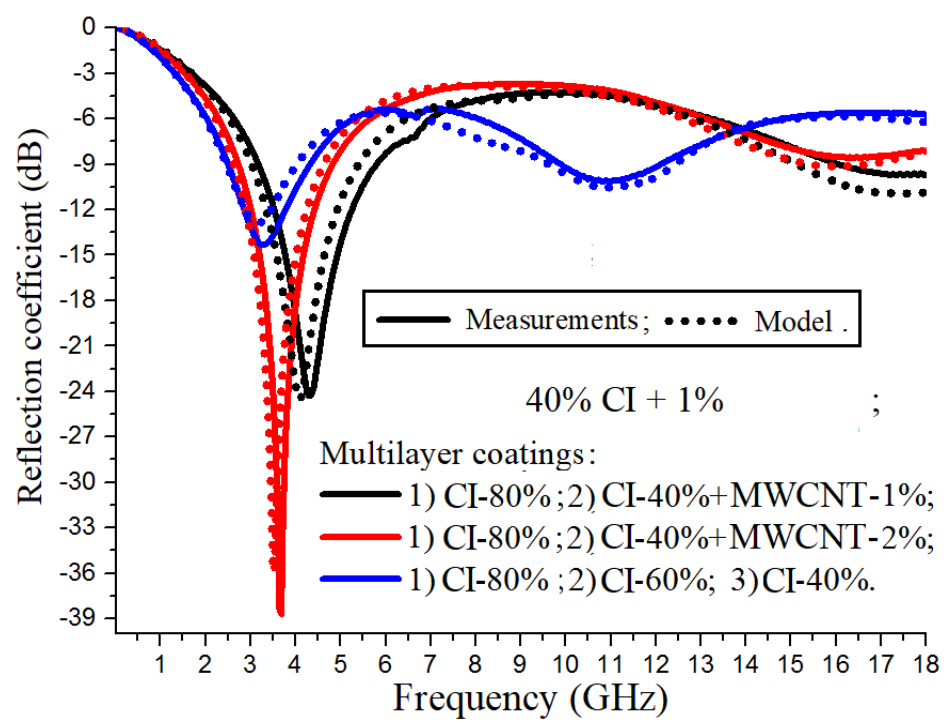

Fig. 6. Frequency dependence of the reflection coefficient from multilayer coatings with different concentrations of carbonyl iron and multi-walled carbon nanotubes.

The measured and calculated values of the reflection coefficient from the composite layer on the metal are compared. They showed a good match. The obtained dependences make it possible to characterize a composite material based on epoxy with the addition of carbonyl iron, multi-walled carbon nanotubes, and barium titanant as effective narrow-band absorbers of electromagnetic radiation in various frequency ranges.

The research was supported by The Tomsk State University competitiveness improvement programme. 


\section{References}

1. B. Quan, X. Liang, G. Xu, Y. Cheng, Y. Zhang, W. Liu, G. Ji, Y. Du, New J. of Chem., 41, 1259 (2017)

2. L. Wangchang, L. Junjun, Z. Xiang, Z. Jingwu, Y. Yao, Q. Liang, Y. Jing, C. Shenglei, $J M M M, 426,504$ (2017)

3. H. Tran, T. Le, B. Pejcinovic, J. Brown, R. Doneker, K. Thompson, IEEE Sym. on EMC, SI \& PI, 41 (2018)

4. L. Liu, L. Kong, W. Yin, S. Matitsine, IEEE Trans. on Elect. Comp., 53, 943 (2011)

5. J. Xiang, Z. Hou, X. Zhang, L. Gong, Z. Wu, J. Mi, J. of Al. and Comp., 737, $412(2018)$

6. X. Ren, H. Fan, Y. Cheng, App. Phys. A: Mat. Sci. and Proc., 122, 506-1 (2016)

7. G. E. Kuleshov, Y. V. Zhuravlyova, O. A. Dotsenko, SIBCON 2015 - Proceedings, 7147115 (2015)

8. K. Chalapat, K. Sarvala, J. Li, G.S. Paraoanu, IEEE Trans. on MW. Theor. and Tech., 57, 2257 (2009) 